\title{
Study on flow behaviour in the short-throat-jet type flotation machine
}

\author{
Hongzheng Zhu ${ }^{1,2}$, Jinbo Zhu ${ }^{1, *}$, Alejandro López Valdivieso ${ }^{2}$, Shaoxian Song ${ }^{3}$ \\ and Fanfei Min ${ }^{1}$ \\ ${ }^{1}$ School of Materials Science and Engineering, Anhui University of Science and Technology, Huainan, 232001, China \\ ${ }^{2}$ Surface chemistry lab, Instituto de Metalurgia, Universidad Autonoma de San Luis Potosi, Av. Sierra Leona 550, San Luis Potosi, \\ SLP 78210, Mexico \\ ${ }^{3}$ School of Resources and Environmental Engineering, Wuhan University of Technology, Wuhan, 430000, China
}

A two-dimensional model of the short-throat-jet flotation machine was developed for studying the flow behaviour and some difficult to measure parameters in the device using computational fluid dynamics (CFD). A laboratorial model was established for verifying the dependability of CFD, while a good agreement between measurement results and simulation was presented. With the inlet pressure set as $0.015 \mathrm{MPa}$, the minimum pressure close to the wall of the inhale section was obtained as $1200 \mathrm{~Pa}$ at $Y=164.2 \mathrm{~mm}$, which was the optimal position for inhaling air. Moreover, the maximum pressure at the cell bottom was about $44,600 \mathrm{~Pa}$ at $X=15 \mathrm{~mm}$ from the centre of the jet, which was useful in selecting a suitable material to build the cell bottom. Analysis of wall shear stress showed that the wall shear stress on the right side of the jet was larger than that on the left side. Closer to the jet nozzle, the shear stress was larger. The wall shear stress on the right side of the short throat was likewise found to be larger than that on the left side, maximum difference being $140 \mathrm{~Pa}$ at $Y=115.5 \mathrm{~mm}$. Analysis of the velocity field showed that the velocity decreased by $50 \%$ from the inlet to the outlet of the throat, and seven obvious vortexes were found to exist in the cell, where two of them in the inhale section were advantageous for inhaling air.

Keywords: Flotation machine, pressure, velocity, vortex, wall shear stress.

SHORT-THROAT-JET type flotation machine is widely used for the flotation of fine minerals like sulphide, coal, etc. $^{1,2}$. This flotation machine exhibits not only a good performance on energy consumption, but also a high sensitivity to slurry concentration ${ }^{3,4}$, which is mainly determined by the hydrodynamic characteristics in the machine; hence it is meaningful to reveal the flow behaviour in the short-throat-jet type flotation machine.

Numerous studies on similar flotation machines have been conducted. Using particle image velocimetry (PIV) system, Wang ${ }^{5}$ studied the flow field in the laboratory model of the jet flotation machine, and several important

\footnotetext{
*For correspondence. (e-mail: zhujinbo1000@gmail.com)
}

circulations in the cell were described. Zhou ${ }^{6}$ explored the flow injection in the aeration device of the FJC (A) type flotation machine, and the equation fitting the liquid level and air intake was obtained. Duan ${ }^{7}$ studied the effects of structural parameters of different impellers on the hydraulic parameters in a simple jet flotation machine model, and the optimal impeller shape and structural parameters were acquired. Until now few studies on the internal flow behaviour have been carried out, and it is important to point out that some difficult-to-measure but important parameters such as the wall shear stress, internal pressure, etc. of the short-throat-jet type flotation machine have not yet been studied.

Computational fluid dynamics (CFD) has been proved to be a dependable technique to study the flow behaviour in flotation machines. Using CFD, $\mathrm{Koh}^{8}$ studied the effect of impeller speed on the air flow behaviour in a selfaerated Denver laboratory flotation cell. Fayed ${ }^{9}$ studied the effects of bubble size on air holdup and hydrodynamics in Wemco pilot cells and predicted the mineral recovery rate of the flotation cell.

The overall aim of this study was to provide a better understanding of the hydrodynamic characteristics in the short-throat-jet type flotation machine. Specific aims were to: (1) verify the dependability of CFD on the study on the short-throat-jet type flotation machine via PIV; (2) reveal the flow regime in the machine; and (3) explore the distributions of velocity, pressure and wall shear stress.

\section{Experiment}

\section{System}

Due to the centrosymmetric structure, quarter of the short-throat-jet type flotation machine was selected for this study. The schematic of the model is presented in Figure $1 a$. Cartesian co-ordinates were established for the model with the centre of the cell bottom as the origin. The main components are: (1) a pipe for water flow, (2) a buffer section, (3) a jet, (4) an inhale section, and (5) a throat $^{10}$. 

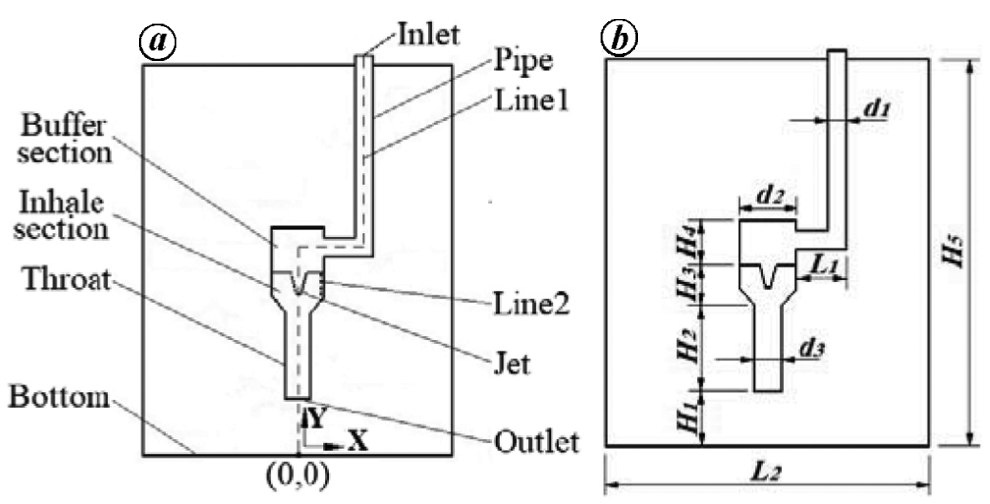

Figure 1. Schematic of the model.

Table 1. Detailed dimensions of the model

\begin{tabular}{lccccccccccc}
\hline Parameter & $H_{1}$ & $H_{2}$ & $H_{3}$ & $H_{4}$ & $H_{5}$ & $L_{1}$ & $L_{2}$ & $d_{1}$ & $d_{2}$ & $d_{3}$ \\
\hline Value/mm & 52 & 80 & 37 & 42 & 360 & 47.5 & 300 & 17.5 & 52 & 26 \\
\hline
\end{tabular}

Water enters the model through the pipe and forms a high-speed stream via the buffer section and jet. Under the effect of high-speed stream, low pressure develops in the inhalation section and air can be inhaled. Water mixes with air in the throat and the mixture flows towards the model bottom ${ }^{11}$. Two lines of interest are defined for the outspread study: line 1 is the symmetric line from the inlet to the bottom of the short throat for which the flow behaviour is important to analyse, and line 2, close to the vessel wall of the inhale section, for which the optimal inhale point is important to analyse.

The model was designed at a scale of $5: 1$ with the industrial machine. The detailed dimensions of the model are given in Table 1 according to Figure $1 b$.

$A$ jet of $5 \mathrm{~mm}$ inner diameter and $19 \mathrm{~mm}$ depth was fixed at the bottom of the buffer section. The length of line 1 and line 2 were 440 and $22 \mathrm{~mm}$ respectively.

\section{Measuring method}

The PIV system (Microvec Pte Ltd, China) mainly consists of four parts $-\mathrm{Nd}$ : YAG dual pulse laser, a synchronizer, a $2400 \times 2100$ pixel $^{2}$ charge-coupled device camera and a Micro Vec V3 software. A software named Microvec contacting the synchronizer was used for controlling all devices connected to the synchronizer and also for analysing the results ${ }^{12-14}$. Because of the use of vitreous throat, the velocity distribution from the jet to the bottom was measured via PIV. Tap water was used as the liquid phase, and metallic oxide particles with diameter of $500 \mathrm{~nm}$ were used as trace particles for visualizing the flow behaviour. The temperature was detected at $25 \pm 1{ }^{\circ} \mathrm{C}$, and necessary pre-calibrations were carried out $^{15}$. At least 300 images of instantaneous velocity field were captured for exploring the representative velocity distribution on the central line of the throat ${ }^{16}$.

\section{Numerical method}

CFD system includes three softwares: (1) Gambit is used for modelling the object, (2) Fluent is used for simulating the model, and (3) Tecplot is applied to visualize the simulation and process the results ${ }^{17-19}$.

For only water as the liquid phase in the flow and assuming no heat transfer, the governing equations are as follows:

Continuity equations

$$
\frac{\partial \rho}{\partial t}+\frac{\partial}{\partial x_{i}} \cdot\left(\rho u_{i}\right)=S_{\mathrm{m}}
$$

Momentum equations

$$
\frac{\partial\left(\rho u_{i}\right)}{\partial t}+\frac{\partial\left(\rho u_{i} u_{j}\right)}{\partial x_{j}}=-\frac{\partial p}{\partial x_{i}}+\frac{\partial \tau_{i j}}{\partial x_{j}}+\rho g_{i},
$$

where $\rho$ is the water density, $t$ the time, $S_{m}$ the extra mass input to the continuous fluid, $u_{i}$ the component velocity at the $i$ direction, $u_{j}$ the component velocity at the $j$ direction, $p$ the water pressure, $g_{i}$ the volume force $\mathrm{e}^{20-23}$ and $\tau_{i j}$ is the term of the viscous stress ${ }^{24}$, which is directly proportional to the deformation rate of the liquid. This can be calculated from eq. (3).

$$
\tau_{i j}=-p \delta_{i j}+\mu\left(\frac{\partial u_{i}}{\partial x_{j}}+\frac{\partial u_{j}}{\partial x_{i}}\right)-\frac{2}{3} \mu \frac{\partial u_{l}}{\partial x_{l}} \delta_{i j}
$$

where $\mu$ is the water viscosity, $\partial \mu_{l} / \partial x_{l}$ the change rate of control volume with time, $\partial / \partial x_{l}$ three dimensional space derivative and $\delta_{i j}$ is the Kronecker delta. 
Table 2. Detailed boundary conditions

\begin{tabular}{lccccc}
\hline Parameter & Fluid density & Fluid viscosity & Operating pressure & Inlet pressure & Outlet pressure \\
\hline Value & $1000 \mathrm{~kg} / \mathrm{m}^{3}$ & $0.001 \mathrm{~kg} /(\mathrm{m} \mathrm{s})$ & $101,325 \mathrm{~Pa}$ & $0.015 \mathrm{MPa}$ & $0 \mathrm{~Pa}$ \\
\hline
\end{tabular}

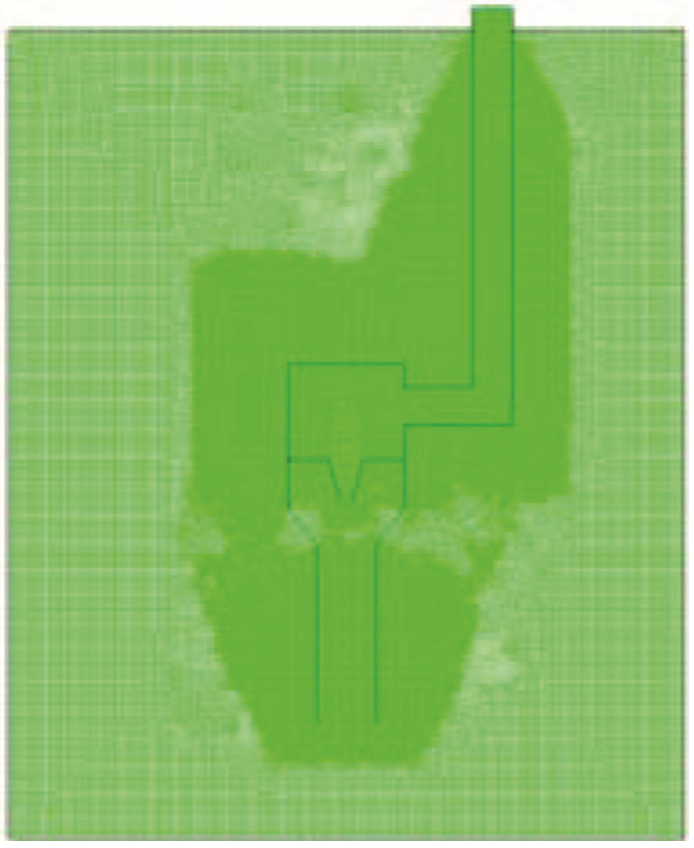

Figure 2. Mesh of the model.

In this study, Gambit 2.4.6 (Ansys Inc., US) was used for modelling and meshing the studied model ${ }^{25}$. A twodimensional model was built with about 56,084 cells as shown in Figure 2. The detailed boundary conditions are provided in Table 2 .

The operating pressure was equal to the atmospheric pressure. The semi-rational formula was used to solve the connection between the vessel walls and the nearby area.

The meshed model, imported into Fluent 6.3.13 (Ansys Inc., US), was simulated using monophasic and standard k-epsilon model. The governing equations were discretized in a non-uniform structural mesh and solved through a single precision mode using finite volume method (FVM). The pressure and velocity were coupled with the SIMPLE algorithm. The residual was set at the fifth-order to ensure simulation convergence ${ }^{22,26}$.

\section{Results and discussion}

\section{Analysis on the velocity distribution}

The velocity distributions, respectively obtained from PIV and CFD, on line 1 were analysed using Tecplot as shown in Figure 3. It should be pointed out that the position represents the distance away from the origin.

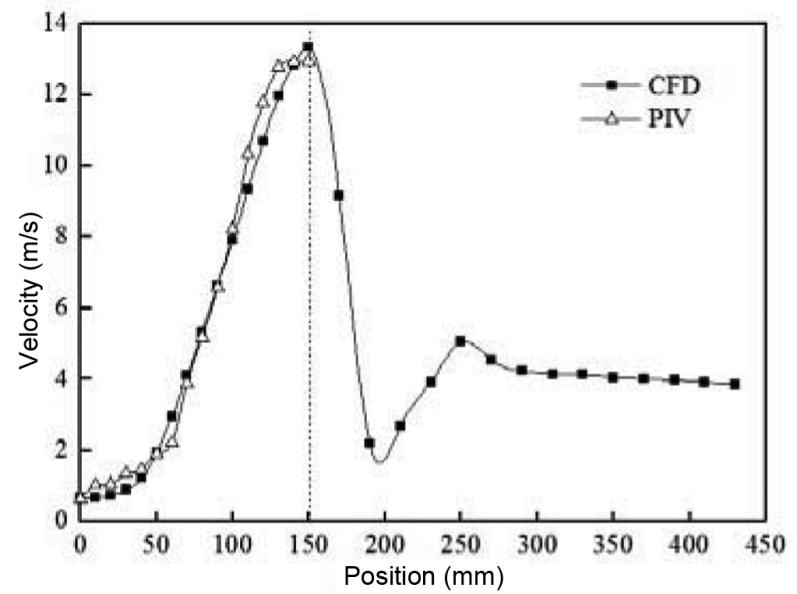

Figure 3. Velocity as a function of position on line 1.

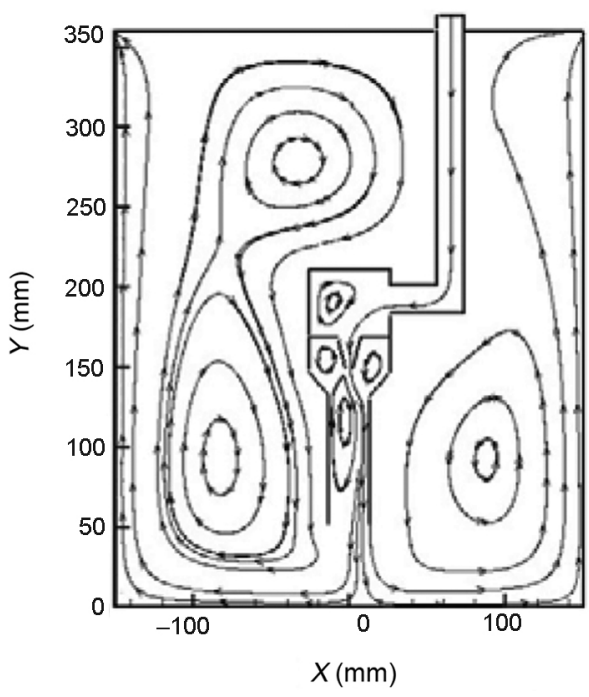

Figure 4. Fluid streamlines in the machine.

It was obvious that the velocity sharply decreased as the position decreased from $150 \mathrm{~mm}$ to 0 , and the PIV curve agreed well with the CFD curve. The variance between these two curves was calculated as approximately 5.56, indicating that CFD was dependable for researching the flow behaviour in this short-throat-jet type flotation machine.

The velocities of the inlet and outlet were measured to be $3.84 \mathrm{~m} / \mathrm{s}$ and $1.93 \mathrm{~m} / \mathrm{s}$ respectively, thus the velocity decreased by $50 \%$ from the inlet to the outlet. The maximum velocity was found to be $13.31 \mathrm{~m} / \mathrm{s}$ at the jet nozzle; therefore the velocity decreased by $85.50 \%$ from the nozzle to the outlet. 


\section{Analysis on the flow regime}

Figure 4 shows the typical streamlines of the fluid. As noted, the flow field was asymmetric, which might be due to the fact that the inlet was on the right side of the symmetry axis of the cell. At least seven obvious vortexes were found in the cell, where two of them in the inhale section were beneficial for inhaling air because of their entrainment effect. Three vortexes were formed in the cell and one in the buffer section. The three vortexes in the cell were detrimental to the flotation process, because they caused circulating movement of particles. As observed, the section of the throat was partly filled, which resulted in the vortex in the throat.

\section{Analysis on the pressure distribution}

The total pressure on the model bottom is given in Figure 5. The pressure distributed as a parabola. The largest pressure was about $44,600 \mathrm{~Pa}$ at $X=15 \mathrm{~mm}$, which was useful for selecting a suitable material to build the cell bottom. Moreover, the minimum pressure was about 39,000 $\mathrm{Pa}$ on the left side of the bottom.

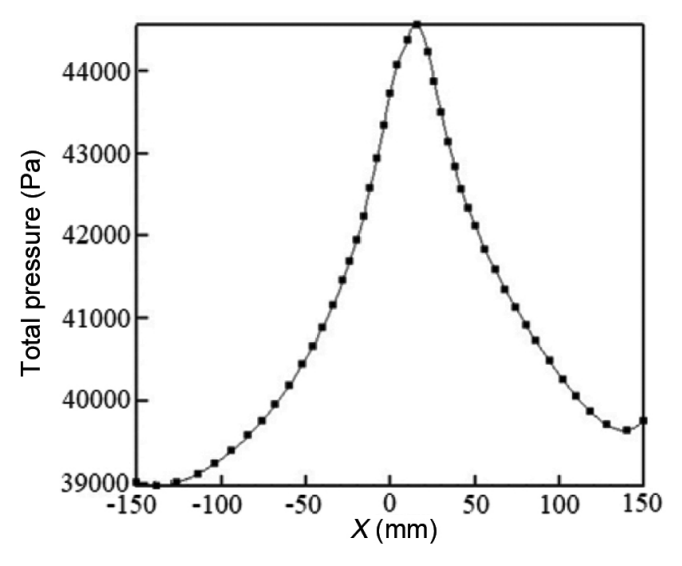

Figure 5. Pressure distribution on the model bottom.

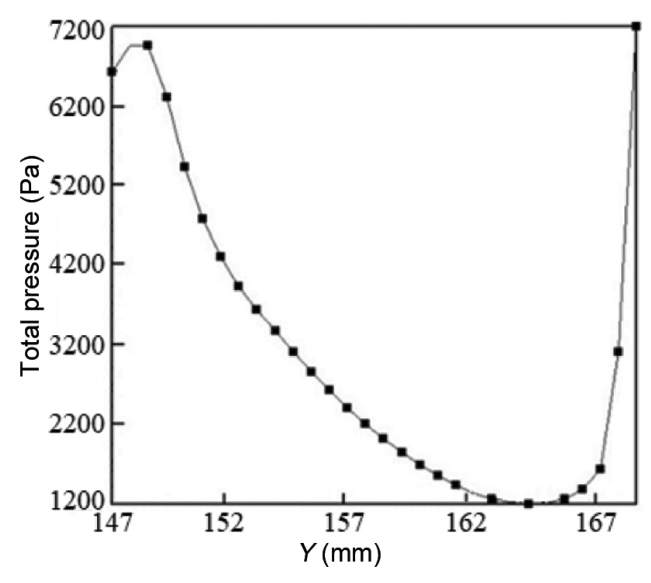

Figure 6. Pressure distribution in line 2.
The total pressure distribution in line 2 is shown in Figure 6. As noted, the minimum pressure was about $1200 \mathrm{~Pa}$ at $Y=164.2 \mathrm{~mm}$, which was smaller than the atmospheric pressure, this being the optimal position for inhaling air.

\section{Analysis on the wall shear stress}

The wall shear stress in the throat is shown in Figure 7. As noted, the trends of the wall shear stress on the left and right sides in the throat were same as those that increased first and then decreased. The maximum wall shear stress was about $250 \mathrm{~Pa}$ at $Y=115.5 \mathrm{~mm}$ on the right side of the throat. The wall shear stress on the right side was larger than that on the left side. When the value of $Y$ is smaller than 98, the difference of the wall shear stress is almost constant about $110 \mathrm{~Pa}$. During the $Y$ from 98 to 115.5 , the difference between these two curves gradually increased from $110 \mathrm{~Pa}$ to $140 \mathrm{~Pa}$. While the $Y$ was larger than 115.5 , the difference was smaller than $140 \mathrm{~Pa}$. An intersection of these two curves was observed at $Y=127.5 \mathrm{~mm}$ and $Y=132.5 \mathrm{~mm}$.

Figure 8 shows the wall shear stress on the left and right sides of the jet. The trends of the wall shear stresses

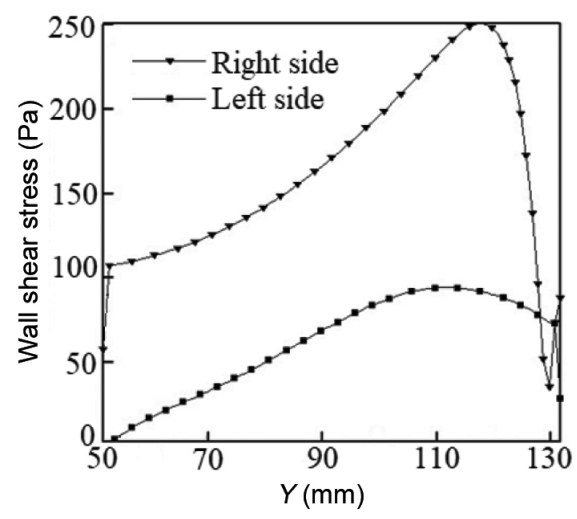

Figure 7. Wall shear stress of the throat.

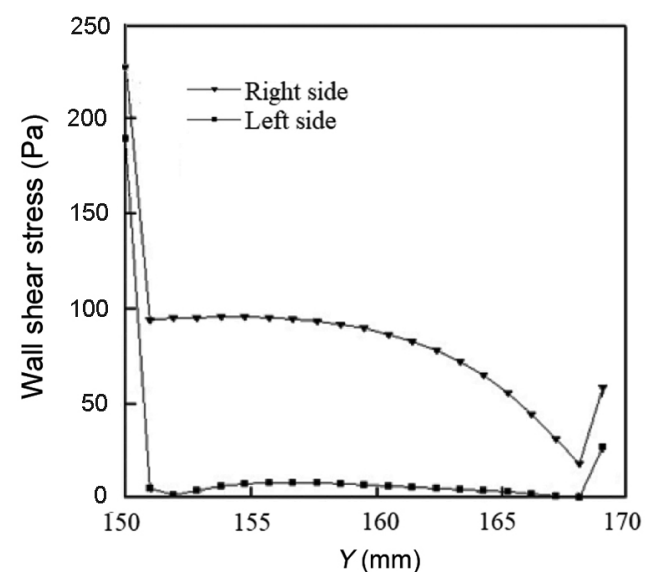

Figure 8. Wall shear stress of the jet. 


\section{RESEARCH ARTICLES}

on the left and right sides were also the same; closer to the jet nozzle, the larger the wall shear stresses were. The maximum wall shear stress on the right side was approximately $230.50 \mathrm{~Pa}$, and that on the left side was $189.55 \mathrm{~Pa}$. The wall shear stress on the right side was larger than that on the left side, which was mainly due to the position of the inlet. This behaviour can be deemed to be a design limitation, which may lead to an easier abrasion on the right side. The difference of the wall shear stress tended to decrease gradually, and the maximum difference was about $90 \mathrm{~Pa}$ at $Y=152 \mathrm{~mm}$.

\section{Conclusion}

In this study, the short-throat-jet flotation machine was experimentally and numerically studied using PIV and CFD respectively. The dependability of CFD used in flow behaviour research in the short-throat-jet flotation machine was assessed. The flow regime, wall shear stress and pressure distribution in the machine were explored. Several conclusions and recommendations can be drawn as follows: (1) Satisfactory agreement between PIV and CFD results testifies the dependability of CFD for flow behaviour research in the short-throat-jet flotation machine. (2) The velocity decreased $50 \%$ from the inlet to the outlet and $85.50 \%$ from the jet to the outlet. Moreover, seven obvious vortexes were found in the cell, where two vortexes in the inhale section were advantageous for inhaling air. The optimal inhale-air position in the inhale section was analysed at $Y=164.2 \mathrm{~mm}$, where the pressure was about $1200 \mathrm{~Pa}$ which is below the atmospheric pressure. (3) The maximum pressure on the bottom was about $44,600 \mathrm{~Pa}$ at $X=15 \mathrm{~mm}$, which was useful for selecting a suitable material to build the cell bottom. The wall shear stress on the right side of jet and throat was larger than that on the left side, which could be deemed as a design limitation that the inlet was designed on the right side.

1. Bakalarz, A., Duchnowska, M. and Pawlos, W., Influence of hydrodynamics on preflotation process in flotation machine. Miner. Metall. Process., 2018, 35, 19-23.

2. Peng, Y., Xia, W., Xie, G. and Yang, H., Coal flotation to satisfy coal quality. Filtr. Sep., 2017, 54, 42-44.

3. Kang, J. et al., A significant improvement of scheelite flotation efficiency with etidronic acid. J. Clean. Prod., 2018, 180, 858-865.

4. Johnson, N. W., Existing opportunities for increasing metallurgical and energy efficiencies in concentrators. Miner. Eng., 2018, 118, 62-77.

5. Wang, H., Study on fluid dynamics and modeling of jet flotation machine. Anhui University of Science and Technology, Huainan, 2010

6. Zhou, W., The study of air mixing device of FJC(A) flotation machine to impact flow injection and experimental. Anhui University of Science and Technology, Huainan, 2011.

7. Duan, Y., Selection and research on parameters of new type flotation machine impeller. Anhui University of Science and Technology, Huainan, 2013.

8. Koh, P. T. and Schwarz, M. P., CFD model of a self-aerating flotation cell. Int. J. Miner. Process., 2007, 85, 16-24.
9. Fayed, H. and Ragab, S., Numerical simulations of two-phase flow in a self-aerated flotation machine and kinetics modeling. Minerals, 2015, 5, 164-188.

10. Zhu, H., Research on structure optimization and large-scale of jet flotation machine. Anhui University of Science and Technology, Huainan, 2012.

11. Zhu, J., Zhu, H., Wang, H., Wu, D. and Zhou, W., Research on hydrokinetics of pneumatic agiator of FJCA20 flotation machine. Coal Prep. Technol., 2012, 5, 18-21.

12. Asgharpour, A., Zahedi, P., Khanouki, H. A., Shirazi, S. A. and McLaury, B. S., Experimental and numerical study on solid particle erosion in elbows mounted in series. In ASME 2017 Fluids Engineering Division Summer Meeting, American Society of Mechanical Engineers, 2017, pp. 15-27.

13. Raghav, V., Sastry, S. and Saikrishnan, N., Experimental assessment of flow fields associated with heart valve prostheses using particle image velocimetry (PIV): recommendations for best practices. Cardiovasc. Eng. Technol., 2018, 9(3), 1-15.

14. Ryu, J.-D., Ha, K.-N., Lee, D.-G. and Nam, K.-S., Flow analysis of underwater water jet system using computational fluid dynamics and particle image velocimetry. Proc. Eng. Technol. Innov., 2017, 6, 23-27.

15. Zhu, H., Song, S., López Valdivieso, A., Zhu, J. and Wang, H., Effects of rectifying bundles on desliming ponds. Int. J. Coal Prep. Util., 2018, 2, 1-10.

16. Day, S. W., Higham, T. E., Cheer, A. Y. and Wainwright, P. C., Spatial and temporal patterns of water flow generated by suctionfeeding bluegill sunfish Lepomis macrochirus resolved by particle image velocimetry. J. Exp. Biol., 2005, 208, 2661-2671.

17. Sivan, K. and Pandian, S., An overview of reusable launch vehicle technology demonstrator. Curr. Sci., 2018, 114, 38-47.

18. Kumar, P., Mishra, D. P., Panigrahi, D. C. and Sahu, P., Numerical studies of ventilation effect on methane layering behaviour in underground coal mines. Curr. Sci., 2017, 112, 1873-1881.

19. Abrahamm, P. K. and Valsa, B., Quality assurance challenges in testing and evaluation of reusable launch vehicle systems. Curr. Sci., 2018, 114, 144-147.

20. Parhi, A. et al., Development of slow-burning solid rocket booster for RLV-TD hypersonic experiment. Curr. Sci., 2018, 114, 74-83.

21. Rabe, B. K., Najafabadi, S. H. G. and Sarkardeh, H., Numerical simulation of air-core vortex at intake. Curr. Sci., 2017, 113, 141-147.

22. Raja Bose, J., Godson Asirvatham, L., Kumar, N., Michael, T. and Wongwises, S., Numerical study on convective heat transfer characteristics of silver/water nanofluid in minichannel. Curr. Nanosci., 2017, 13, 426-434.

23. Vidya, G. et al., Aerodynamic design, characterization and flight performance of RLV-TD. Curr. Sci., 2018, 114, 48-63.

24. Zhang, Y. et al., Computational fluid dynamics study on mixing mode and power consumption in anaerobic mono-and codigestion. Bioresour. Technol., 2016, 203, 166-172.

25. Yang, Q., Lv, W., Ma, L. and Wang, H., CFD study on separation enhancement of mini-hydrocyclone by particulate arrangement. Sep. Purif. Technol., 2013, 102, 15-25.

26. Ross, J. and Al-Shahi Salman, R., The frequency of thrombotic events among adults given antifibrinolytic drugs for spontaneous bleeding: systematic review and meta-analysis of observational studies and randomized trials. Curr. Drug Saf., 2012, 7, 44-54.

ACKNOWLEDGEMENTS. We acknowledge the Provincial University Natural Science Foundation of Anhui (Grant No. KJ2016A188), National Natural Science Foundation of China (Grant No. 51374015), and National Council of Science and Technology of Mexico (CONACyT) for the Fellowship No. 742903 to carry out this work.

Received 3 October 2017; revised accepted 5 November 2018

doi: $10.18520 / \mathrm{cs} / \mathrm{v} 116 / \mathrm{i} 4 / 592-596$ 